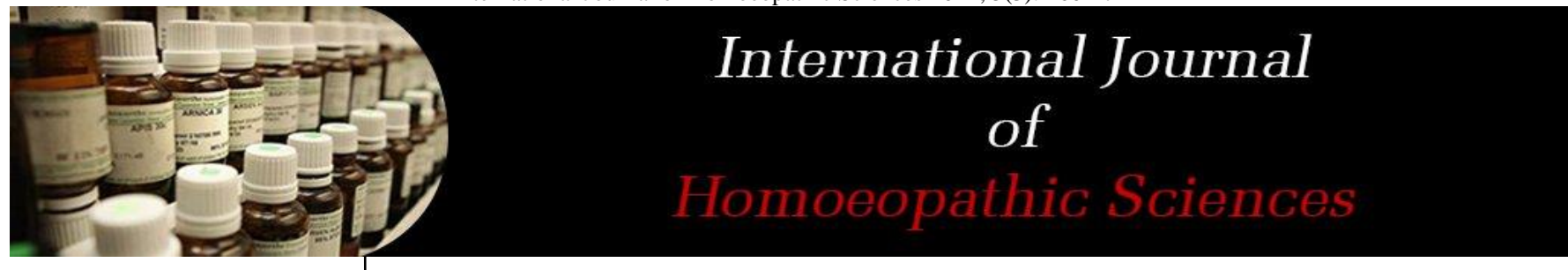

E-ISSN: $2616-4493$ P-ISSN: 2616-4485 www.homoeopathicjournal.com IJHS 2021; 5(3): 160-171 Received: 22-05-2021

Accepted: 24-06-2021

Chavan RB

Retired Professor, Indian Institute of Technology Delhi, Delhi, India
Corresponding Author:

Chavan RB

Retired Professor, Indian

Institute of Technology Delhi, Delhi, India

\title{
Homoeopathy: It's role in palliative and curative care of cancer
}

\section{Chavan RB}

DOI: https://doi.org/10.33545/26164485.2021.v5.i3c.421

\begin{abstract}
An attempt has been made to briefly describe the origin of Homoeopathy, its advent in India, global acceptance, safety, limitations and evolution of Banerji Protocols.

The major focus of the present review is on the role of homoeopathy in palliative and curative care of cancer. The findings from several laboratories and clinical studies suggest that homeopathy might have some beneficial effect in cancer care. In animal models, several homeopathic remedies showed an inhibitory effect on certain tumor development. Some clinical studies of homeopathic remedies combined with conventional care have shown that homeopathic remedies reduce the burden of side effects after conventional surgery, chemo and radiation therapies and thus improve quality of life and in some cases improve survival of cancer patients. However, further comprehensive clinical studies are warranted to confirm these beneficial effects. Considering the low cost, minimal risks, and the potential beneficial effects, the use of homoeopathy might be considered as an additional tool to integrate as complementary alternate medicine (CAM) with allopathic science for cancer care.
\end{abstract}

Keywords: homoeopathy, palliative, cancer, apoptosis, Ruta graveolens, sarsaparilla, Carcinosin

\section{Introduction}

The word homoeopathy is derived from the Greek words "Omosis" means similar and "Pathos" means suffering. According to some resources, it is a technique used by Hippocrates who was an ancient Greek physician in $5^{\text {th }}$ century BC, sometimes he is known as father of medicine.

It is believed that, Hippocrates treated mania (madness or insanity) by administering small doses of the mandrake root which in large doses is known to trigger mania. (Mandrake is the root obtained from plants of genus Mandragora found in Mediterranean region or from other species such as Bryonia alba known as English mandrake which have similar properties ${ }^{[1]}$.

The present medical science of homeopathy was developed about 230 years ago by the German physician Samuel Hahnemann (1755-1843). When Dr. Hahnemann tested quinine (used in healing malaria) on himself in 1789, he observed the emergence of malaria-like symptoms. Dr. Hahnemann continued to perform similar tests on people in his immediate neighbourhood, and then put forward his new doctrine of "Like cures like" that constitutes the basis of homeopathy ${ }^{[2]}$.

This is exactly opposite of the modern allopathy medical science that treats disease symptoms by medicines that produce opposite effect.

In consequence of his experiments and observations, Dr. Hahnemann put forward the following the most important fundamental principles of homoeopathy.

- A diluted remedy provides healing when given to a patient showing symptoms that appear when the same remedy is given to a healthy person. (Like cures like).

- Homeopathic remedies are first tested on healthy people, before introducing to use for patients.

- One homoeopathic drug may be used for treating more than one illness.

- The remedy is potentised for ensuring the minimal concentration of the active ingredient. (Potentisation means to make homoeopathic medicine more powerful by successive dilutions and shaking.)

- When potentised (diluted), the remedy becomes more effective. i.e. dilute the remedy more effective it is. 
Dr. Hahnemann believed that the potentisation process reveals the spiritual power of the medicine and eliminates its harmful effects.

Hahnemann also emphasized the evaluation of patient holistically based on "individualization" and "similimum".

"Individualization" means associating illness with emotional, mental, physical health and environmental conditions of patient. According to this principle each patient is unique and the choice of medicine for a particular illness will depend on the unique individual features of the patient and the medicine. In conventional medical practice only symptoms of illness are considered for making the choice of medicine.

"Similimum" means the most similar (appropriate) medicine according to the specific features of patient (individualization) and his/her disease symptoms.

Dr. Hahnemann explained the principles of classical homoeopathy in his book "The Organon of the Medical Art" published in 1810 . The book is used as a guide in education and practice of homoeopathy ${ }^{[3,4]}$

Homeopathy gained popularity especially in the 19th century because during epidemics experienced in that period, the death rates in homeopathy hospitals were lower. It remained in the background in the 20th century as a result of the developments in modern allopathy medical science. However, homoeopathy began to regain popularity in the late 20 th and early 21 st centuries ${ }^{[5]}$.

According to the 2012 U. S. National Health Interview Survey (NHIS); 5 million adults and 1 million children used homeopathic medicines per year. When this result was compared to the results of the 2002 and 2007 surveys, it was observed that the number increased with each passing year $[6,7]$.

Another national survey published in 2016 reported that $2.1 \%$ of adults in the U.S. used homeopathic products in the year $2015^{[8]}$

Homoeopathic medicines are the extracts of herbal, animal and mineral origin. In general, with few exceptions, homoeopathic medicines are claimed to be harmless i.e. without any side effects based on the fact that the active ingredients contained in homeopathic products are highly diluted except for rare and non-serious side effects such as allergic reactions.

The diseases most commonly treated by homoeopaths are asthma, depression, allergic rhinitis, migraine, neurotic disorders, non-specific allergies, dermatitis, arthritis and hypertension.

\section{History of Homoeopathy in India}

The history of the advent of homoeopathy in India is quite fascinating. As early as 1810, few German missionaries landed in Bengal. They used to distribute homeopathic medicines among the poor people to alleviate their sufferings.

Looking at the success of these German missionaries due to the efficacy and affordability of homoeopathy medicines in the rural environment, many school teachers took to reading the homeopathic Materia Medicas (A well known book of homoeopathy medicines) and started prescribing to village communities ${ }^{[9]}$.

John Martin Hoenigberger from France took education in Homoeopathy under the guidance of Dr. Hahnemann in 1835. Hoenigberger visited India in 1852 and started practicing homoeopathy in Lahore at the court of Maharaja
Ranjit Singh. Subsequently he wrote a book providing a vivid account of his successful treatment of Maharaja Ranjit Singh's chronic disease of partial paralysis. This book gave glimpses of the beginning of homoeopathy in India ${ }^{[10]}$.

During the second half of the nineteenth century few homeopathic dispensaries were opened in Bengal and in the south. The pioneer in this field in Calcutta was Rajendralal Dutta (1818-1889). He belonged to a scholarly and aristocratic family of Bengal. He engaged a French doctor, Dr. Tonnere, and placed him in charge of a homeopathic dispensary in Calcutta. Unfortunately, this venture failed. Subsequently, Rajendralal Dutta himself took up the responsibility and started practice in homeopathy. Among his illustrious patients may be mentioned the great early social reformer Pandit Ishwarchandra Vidyasagar and Raja Radhakanta Dev Bahadur. Rajen Dutta cured Pandit Vidyasagar of a migraine where conventional system had failed. Furthermore the cure of a gangrenous ulcer of Raja Radhakanta Dev Bahadur created a sensation in Calcutta at that time.

In order to strengthen the roots of homeopathy practice in India, Dutta looked around for a suitable person of eminence. He persuaded Dr. Mahendralal Sircar, (medical doctor and skeptic of homeopathy) to test scientifically the efficacy and curative potentials of homoeopathy medicines. In his experiments; administration of homeopathic medicines became effective even when Dr. Sircar's conventional medicine failed. Thus Dr. Sircar converted himself to homeopathy and carved a niche for it in the medical history of India. A number of allopathic doctors started homeopathic practice following Dr. Sircar's lead.

'The Calcutta Homeopathic Medical College', the first homeopathic medical college, was established in 1881. This institution took on a major role in popularizing homeopathy in India ${ }^{[11]}$.

In this context it is worth mentioning the work of Dr Pareshnath Banerji in popularising homoeopathy in Bengal.

Late Dr. Pareshnath Banerji started his charitable clinic in a remote village, Mihijam, situated in the border of Bihar in 1918 and soon became a legend. He achieved phenomenal success against all kinds of disease as a result there was continuous flow of patients to his clinic.

In order to cope up with flow of patients every day he did not always adhere to Hahnemann's Dictum of classical homoeopathy. Also homoeopathic medicines in the form of mixtures and frequent repetition of medicines were prescribed when required. This was the basis of Banerji protocols described later ${ }^{[9]}$.

Gradually homeopathic dispensaries opened in other cities like Benares and Allahabad, and by the beginning of the twentieth century homeopathy had spread all over India. In 1973, the Government of India recognized homeopathy by setting up the Central Council of Homeopathy $(\mathrm{CCH})$ to regulate its education and practice ${ }^{[11]}$.

Now, only qualified registered homeopaths can practice homeopathy in India. Presently; Ayush a separate ministry is set up by Govt. of India to encourage scientific studies on all traditional medical practices including Homoeopathy. In India, homeopathy is the third most popular system of medicine (after Allopathy and Ayurveda), due to the fact of its easy administration in the home setting and its affordability. 


\section{Limitations of classical homoeopathy and evolution of Banerji protocols}

Although homoeopathy is well accepted worldwide, it is associated with few limitations:

\subsection{Difficulty in making choice of medicine}

The homeopathic approach is holistic, that is, while treating a patient a homeopath will consider not only the disease symptoms but the whole constitution of the patient. The patient is treated as a whole. This principle of treatment is known as "individualization" and "similimum"

"Individualization" means every individual patient is physically, mentally, constitutionally and likes and dislikes is different. In general, all individual patients have their own special features.

"Similimum" means the most similar (appropriate) medicine as per the specific features of patient and his/her disease symptoms After noting down the individual features and disease symptoms of a patient, the homoeopath thinks of a few medicines out of which he/she finds one medicine which appears to be the most similar to the symptoms and individual features. In classical homeopathy only a single medicine is given and then the patient is observed for his/her response.

Classical homeopathy has, therefore, no specific remedy for any specific disease by name, but it has specificity for each individual case and disease symptoms. The medicine is selected based on practical experience of the homoeopath e.g. ten cases of Tuberculosis may require ten different medicines, where as ten different disease conditions may require same medicine if there is match between medicine and individual patient features. This means homoeopathic medicines are classified according to individual patient features. Therefore, a specific homoeopathy medicine cannot be used according to disease symptoms.

This procedure is subjective and requires a long and intense interrogation of the patient. For this reason if the same patient is examined by different homeopaths, their opinions in terms of individualization and similimum and the choice of medicine may not match with each other.

\subsection{Earning and experience gaining}

Another practical problem may be mentioned here. If a homeopath examines six or eight cases daily, he may have to charge high fees from each individual patient for maintenance of clinic. This will put homeopathy out of reach to the suffering population. At the same time, with such a small number of patients, a homeopath hardly gets enough clinical experience to become a true physician. It is common knowledge that experience makes a doctor.

\subsection{Limited choice for scientific validation}

Scientific validation of the efficacy of homeopathic medicines, which are nontoxic and inexpensive; making them ideal as "the People's Medicine" has been hindered due to inability to conduct clinical trials using standardized treatment protocols.

The true healing potential of homeopathy, therefore, has been repeatedly challenged and denied by mainstream medical professionals.

\subsection{Evolution of Banerji Protocols}

P Banerji Homeopathy Research Foundation established by Prasanta Banerji and Pratip Banerji at Kolkata (PBHRFI, pbhrfi.org) does not practice the principles of classical homeopathy. The foundation has developed a method of treatment in which specific medicines are prescribed for specific diseases like in Allopathy. Diseases are diagnosed using modern state-of-the-art diagnostic techniques such as CT/PET scans, NMR, Ultrasound, biopsy etc. The efficacy of this approach is reflected by encouraging results in homoeopathy treatments. This new concept of treatment is known as "Banerji Protocols." The protocols also encourage the use of potentized medicines in combination to provide quick and un-eventful (without side effects) recovery in much shorter time ${ }^{[9]}$.

\section{Global acceptance of homeopathy}

Homeopathy currently is used in over 80 countries around the world. In several countries including India, Mexico, Brazil and the UK, homeopathy is integrated into the healthcare systems. In the United States, homeopathy remedies are regulated as non-prescription drugs.

Homeopathy is very popular in the UK, where the Royal Family has homeopathy physicians since 1830. In England, as many as $45 \%$ of conventional MDs refer patients to homeopaths for complementary alternate medicine (CAM) and the treatment is part of the National Health Scheme (NHS).

In France, $40 \%$ of the population, 30 thousand doctors and $32 \%$ of French family physicians use homeopathy as complimentary alternate medicines (CAM)

In Italy there are 20 thousand homoeopathy pharmacies which indicate that homeopathy is the most popular alternative therapy, used by large population.

In Germany $25 \%$ of family physicians use homeopathy, and are reimbursed by the National Health System ${ }^{[9]}$.

In Brazil, nine million people use homeopathy. In 1985, homeopathy was included among the therapeutic options offered at the outpatient facilities of the Brazilian public health system. Fifteen thousand Brazilian doctors practice homeopathy ${ }^{[12]}$

In Asia, the homeopathy is very popular, especially in India, Pakistan and Sri Lanka. The historical statement of Mahatma Gandhi "Homeopathy cures a larger percentage of cases than any other method of treatment and is beyond doubt safer and more economical and most complete medical science," added another feather in the cap of homeopathy. In India there are 162 degree colleges teaching homeopathy and the largest pool of homeopaths in the world. Over 200,000 doctors practice homeopathy. About 100 million people use homeopathy. This is an approximate estimate because in the 1950 s and 1960s there were not many conventional doctors available for the treatment of the masses. In that era, most village school teachers and scholars educated themselves in this economical and easy to administer treatment form ${ }^{[11,13]}$

According to WHO report in 2006, Of India's 1.16 billion populations, approximately $70 \%$ live in villages and rural areas, where access to expensive conventional medical facilities is limited and depend on traditional medical practices including homoeopathy ${ }^{[14]}$.

\section{Safety of homoeopathy medicines}

Homoeopathy medicines are prepared from the extracts of herbs, animals and mineral products. The active ingredients contained in homeopathic products are highly diluted, therefore, they are claimed to be harmless i.e. without any 
side effects, except for rare and non-serious side effects such as allergic reactions ${ }^{[1]}$.

However, few studies reveal that inadequately diluted products containing high levels of active ingredients are sometimes available in the market which may be harmful. Toxic components have also been found in some homeopathic products. After using arsenic containing homeopathic medication, there is possibility of developing gastrointestinal disease ${ }^{[15]}$.

In a study, toxic metal concentrations of homeopathic products sold in Croatia were measured and toxic substance at a hazardous level was found in some products, although the levels were generally below the threshold value ${ }^{[16]}$.

In another case it is reported that excessive use of a homeopathic medication called Lycopodium similiaplex led to acute hepatitis ${ }^{[17]}$.

In the report prepared by the World Health Organization with the participation of 400 experts from more than 105 countries, it was stated that Homeopathic products are generally safe as long as they are prepared properly; however, high concentrations of active ingredients in insufficiently diluted products may cause side effects ${ }^{[18]}$.

In a systematic compilation published in 2012, allergic reactions and intoxications caused by homeopathic medications were reported. Besides their direct effects, homeopathic medications can also be indirectly harmful by causing the main treatments to be received inadequately ${ }^{[19]}$.

\section{Homoeopathy in cancer care}

Allopathic medicines though effective for cancer treatment, they are associated with adverse side effects affecting the quality of life of cancer patient. Some cancer patients choose to incorporate complementary and alternative medicines (CAM) or therapies such as Ayurveda, homeopathy, meditation, acupuncture, yoga, energy healing, naturopathy, hypnosis etc. into their care to improve survival with quality of life. Among these CAM therapies Homoeopathy has gained much importance ${ }^{[20]}$.

The scope of Homeopathy for cancer care varies depending on the type of cancer, stage of cancer and the general health condition of the patient. Following are some of the aspects of Cancer care with Homeopathy:

- One of the most distressing complaints associated with some varieties of Cancer is the agonizing pain. Conventional medicines can provide pain relief but only to a certain extent and these medicines are not without any side effects. Moreover, there is always a restriction to the dosage that can be safely administered to the patient. The advantage of administering Homeopathic medicines in such cases is that there can be effective pain control without inducing any side effects.

- Homeopathy can help in improving the general wellbeing and vitality of the patient.

- Conventional treatment options for cancer (surgery, chemotherapy, radiotherapy, etc) are associated with distressing side effects and homeopathy can play a definite role to counter these side effects.

- The diagnosis of cancer often leaves the patient with a sense of depression, anxiety, and fear. The treatment may induce additional irritability, impatience and mood fluctuations. Homeopathy can influence the psyche of the patient and help him/her to deal with these emotions in a better way.
- Homeopathic medicines may also have a role to play in controlling the progression and spread of the disease to other organs.

Homeopathic medicines can be administered along with the allopathic medicines ${ }^{[21]}$.

A 2015 search of the term homeopathy in the data base PubMed produced over 5000 citations; approximately 250 of these citations relate to homeopathy and cancer. With this increased interest the need to explore the evidence about the benefits of this method of care arose among cancer patients worldwide ${ }^{[22]}$.

In UK, almost $30 \%$ patients in homoeopathy hospitals were referred directly by oncologists.

There is a need to setup a research programme for finding the efficacy of homoeopathic medicine against cancer. Some homeopathic medicines have been proven experimentally and clinically; however, there is a wide range of medicines on which clinical data is not available [23].

A survey of 1000 cancer patients in 14 European countries revealed that $36 \%$ of cancer patients were using some form of complementary medicines among which homoeopathy was prominent ${ }^{[24]}$.

In Germany, Homeopathy is the most frequently used CAM treatment among German adults and children with cancer [25].

In Italy, homeopathy is one of the leading complementary therapies used by cancer patients. One survey at two oncology day hospitals revealed that $17 \%$ of patients on chemotherapy used Homeopathy and herbal medicines ${ }^{[26]}$.

Another European survey published in 2015 involved 236 centres that provide integrative/alternative oncology services in the public health system among which homeopathy was one of the leading CAM therapies ${ }^{[27]}$.

\section{Scientific studies on homoeopathy for cancer care 7.1 Laboratory studies on Mice}

Amri et. al. have investigated in the laboratory the effect of homeopathic remedies on cancer cell survival. Their findings revealed reduction in tumor size in mice that were inoculated with human prostate cancer cells and were treated with the homeopathic remedy of Sabal Serullata. In this study, the examination of the ultra structural cytomorphology revealed cellular disintegration which cannot be explained with the well-defined apoptosis or necrosis of cell death. The analysis indicated a novel caspase-independent cell death, which might explain the significant tumor size reduction in the treated animals.

The experimental data suggest that these ultra low concentrations triggered a pathway not yet characterized as cell death and not related to classical apoptosis or necrosis [28].

MacLaughlin and his group found that prostate tumor was significantly reduced in Sabal Serrulata-treated mice compared to untreated controls. It was concluded that $\mathrm{S}$. Serrulata should be further investigated as a specific homeopathic remedy for prostate cancer ${ }^{[29]}$.

Sunila and Kuttan evaluated the effect of the homeopathic remedy Thuja occidentalis extract on the inhibition of lung metastasis induced by melanoma cells in mice. A marked reduction in tumor-nodule formation was shown. The lifespan of the Thuja-treated mice was also significantly increased ${ }^{[30]}$. 
In an animal model study, the inhibitory effects of potentised preparations of Hydrastis,

Lycopodium, Ruta and Thuja against sarcomas that were induced by 3-Methylcholanthrene as well as hepatocellular carcinoma induced by N'-nitrosodiethylamine in mice was studied. Biochemical, morphological and histopathological evaluations revealed the reduction of marker enzymes and tumour size. Among the four medicines, Ruta 200c was most effective in reducing tumour size and incidence of sarcomas ${ }^{[31]}$.

A homoeopathic medicine, Chelidonium in ultra-low doses showed anti-tumour and anti-genotoxic potential against hepatocarcinoma that was induced by p-dimethyl aminoazobenzene (pDAB) in mice ${ }^{[32]}$.

In another animal study anti tumor and anti metastatic effects of various homeopathic medicines were studied in mice against transplanted tumors. Hydrastis and Ruta significantly increased the lifetime of Carcinoma induced tumour bearing mice. Moreover, Most of the Hydrastis 1Mtreated animals were completely tumour free. Hydrastis $1 \mathrm{M}$, Lycopodium $1 \mathrm{M}$ and Thuja $1 \mathrm{M}$ exhibited anti-metastatic effect in melanoma-bearing animals ${ }^{[33]}$.

P-dimethylaminoazobenzene induced Hepatocarcinogen mice were used to determine the anti-carcinogenicity of Natrum sulph. 200. This medicine showed potentials to reduce the cancer as compared to Natrum sulph $30^{[34]}$.

Korengath Preethi and co-workers used Ruta 200C, Carcinosinum 200C, Hydrastis 200C, Thuja 200C, and Thuja 1M. These drugs were tested on cancer cell induced mice. The effect of homoeopathic medicines on cancer cell apoptosis was measured by microarray analysis. Activity of Ruta 200C was compared with that of the mother tincture. It was concluded that Ruta $200 \mathrm{C}$ produced morphological changes in tumor cells. Carcinosinum 200C increased apoptotic gene p53 and Ruta 200C decreased anti-apoptotic gene $\mathrm{Bcl} 2$. Microarray analysis of cells treated with homoeopathic drugs indicated that many enzymes related to apoptosis were increased by homoeopathic drugs. These data indicated that apoptosis is one of the mechanisms of tumor reduction by homeopathic drugs. A comparison of potentiated drugs with their mother tincture indicated that the potentiated drugs have biological activity similar to that of their mother tinctures ${ }^{[35]}$.

Surjyo Jyoti Biswas and co-workers conducted a study to examine whether the potentized homeopathic remedy Carcinosin 200, fed alone and in combination with Chelidonium 200, has protective effects against pdimethylaminoazobenzene (p-DAB) induced Hepatocarcinogenesis (lever cancer) in mice.

Liver tumors were induced in mice through feeding of $\mathrm{p}$ DAB (initiator) and Phenobarbital

(Promoter). The mice were divided into two subgroups: One was fed potentized Alcohol 200 and served as placebo; and the second was fed Carcinosin 200 alone or in combination with Chelidonium 200.

It was observed that Both Carcinosin 200 and Chelidonium 200 when administered alone showed considerable curative effect against p-DAB-induced lever cancer cells in mice. The combination feeding of these two drugs appears to have had a slightly greater protective

effect.

It was concluded that these homeopathic remedies have the potential to be used as complementary and alternative medicine in liver cancer therapy, particularly as palliative measures ${ }^{[36]}$.

\subsection{Laboratory studies on cancer cell lines}

Shagun Arora and coworkers assessed the cytotoxic activity of selected homeopathic medicines in mother tincture (MT), and different dilutions (30C, 200C, $1 \mathrm{M}$ and 10M) against cell lines deriving from tumors of particular organs, Sarsaparilla (Sars) on (human renal adenocarcinoma), Ruta Graveolens (Ruta) on human colorectal carcinoma, and Phytolaccadecandra (Phyto) on human breast carcinoma. Cytotoxicity was measured using the 3-(4, 5dimethylthiazolyl-2)-2, 5-diphenyltetrazolium bromide method, anti-proliferative activity by Trypan Blue exclusion assay, apoptosis determined by dual staining the cells with ethidium bromide (EB) and Acridine Orange (AO) dyes.

The researchers observed that Mother tinctures and ultradiluted preparations of the three homeopathic medicines had highly significant effects in the respective cancer cells, producing cytotoxicity and a decrease in cell proliferation. The effects were greatest with the Mother tinctures compared to ultra-diluted preparations.

This study provides preliminary laboratory evidence indicating the ability of homeopathic medicines as anticancer agents. Further studies of the action of these homeopathic remedies are necessary ${ }^{[37]}$.

Natural dietary supplements have shown effectiveness in the prevention and treatment of cancer. Sarsaparilla (Smilax Glabra Rhizome) has growth-inhibitory effects on several cancer cell lines in vitro and in vivo, with little toxicity on normal cells. However, the mechanism underlying its function remains elusive. Tiantian She et al examined the anticancer activity of the supernatant of the water-soluble extract (SW) from sarsaparilla. Liquid chromatography/mass spectrometry analysis identified flavonoids, alkaloids, and phenylpropanoids as the major bioactive components of SW. SW was shown to markedly inhibit the growth of a broad spectrum of cancer cell lines in the in vitro and in vivo assays. The mechanism of apoptosis of cancer cells was suggested.

This study provided a theoretical basis for sarsaparilla as an anticancer agent ${ }^{[38]}$.

\subsection{Scientific studies on cancer patients}

Gilles Freyer and co-workers investigated the effectiveness of Ruta Graveolens 9c for improvement in quality of life and tumor progression. 31 Patients (>18-years, lifeexpectancy about 3 months), with locally advanced tumours or metastases, previously treated with all available standard anti-cancer treatments were recruited. Oral treatment consisted of two 1-mL ampoules of Ruta Graveolens (9c dilution) given daily for a minimum of 8 weeks, or until tumour progression.

Primary outcome was QoL (Quality of life) measured using the EORTC QLQC30 questionnaire. Secondary outcome measures were anxiety/depression measured using the Hospital Anxiety and Depression Scale (HADS), WHO performance status (PS).

Tumour progression was assessed using RECIST criteria.

At the end of study, it was concluded that all the patients tolerated Ruta 9c doses, QoL global health status improved significantly; there was no significant change in anxiety/depression or PS during treatment and Ruta Graveolens 9c had no obvious effect on tumour progression. However, further studies are necessary to confirm the 
effectiveness of this treatment ${ }^{[39]}$.

Sen Pathak and co-researchers investigated the brain cancer cell-killing activity of a homeopathic medicine, Ruta Graveolens. They treated human brain cancer and HL-60 leukaemia cells, normal B-lymphoid cells, and murine melanoma cells in vitro with different concentrations of Ruta in combination with $\mathrm{Ca}_{3}\left(\mathrm{PO}_{4}\right)_{2}$

Fifteen patients diagnosed with intracranial tumors were treated with Ruta 6 and $\mathrm{Ca}_{3}\left(\mathrm{PO}_{4}\right) 2$. Of these 15 patients, 6 of the 7 glioma patients showed complete regression of tumors. Normal human blood lymphocytes, B-lymphoid cells, and brain cancer cells treated with Ruta in vitro were examined for telomere dynamics, mitotic catastrophe, and apoptosis to understand the possible mechanism of cellkilling, using conventional and molecular cytogenetic techniques. Both in vivo and in vitro results showed initiation of survival-signalling pathways in normal lymphocytes and initiation of death-signalling pathways in brain cancer cells. Cancer cell death was initiated by telomere erosion and completed through mitotic catastrophe events.

These researchers proposed that Ruta in combination with $\mathrm{Ca}_{3}\left(\mathrm{PO}_{4}\right)_{2}$ could be used for effective treatment of brain cancers, particularly glioma ${ }^{[40]}$.

\section{Evolution of the Banerji Protocols}

The credit of Research in homeopathy in India and introduction of Homeopathic medicines in mixtures goes to the $\mathrm{P}$ Banerji Homoeopathy Research Foundation India (PBHRFI). The foundation was established in 1992 at Kolkata by two renowned homoeopaths, Dr Prasanta Banerji and Dr Pratip Banerji.

The aim of this foundation is to establish homoeopathy as a scientific and effective branch of medicine. The homoeopathy clinics under the foundation provide comprehensive health care to bring homoeopathy to its rightful position as the medicine for masses.

Banerji Protocols are the fruit of a cumulative experience and careful analysis of observed trends in patient - medicine interaction and the translation of the same into a system of prescribing with a view to standardize and make easy the practice of an extremely complex system of medicine using ultra dilute solutions ${ }^{[41]}$.

The use of specific medicines in specific potencies for a specific disease in fixed dosage eliminates the necessity for any guess work on the part of new practitioners and is always a tremendous help for even seasoned doctors. This approach is more diagnostic than individualistic, i.e. more objective than subjective. These protocols are easy to learn and since the focus is on the diagnostic approach the time spent with patients for making a case paper is shortened.

Consequently, it also makes the treatment affordable to the weaker sections of society, making it "The People's Medicine." For any scientific medical system it is a rule that interventions should be repeated with almost the same result - meaning, a treatment should have repeatability and the
Banerji Protocols fulfil this criterion.

\subsection{The Banerji Protocols in the treatment of cancer}

At PBHRFI clinics in Kolkata; an average patient turnout is 1000 to 1200 a day out of which an average of 10 to $15 \%$ of the patient turnout is of cancer cases.

The patients from more than seventy countries followed the Banerji Protocols for treatment of cancer through the website www.pbhrfindia.org, seeking online medical advice and treatment on every type of cancer and every stage of the disease. The majority of patients opt to take only homoeopathy treatment without any conventional treatments. However, there are also patients who use homoeopathy medicines as adjunct therapy along with or after conventional treatments. There are also patients who seek relief from the various side-effects of conventional surgery, chemotherapy and radiation. Banerji protocols for the different types of cancer are mostly customized in terms of medicine choice, dilutions and dosages according to the type of cancer and it's location

The main objective of Banerji Protocols is to provide cancer patients a better quality of life and, if possible, to provide a permanent cure. The Banerji Protocols are designed taking into account the diagnosis as well as the various side effects being suffered by the patients.

The basic set of medicines to treat each cancer type consists of 1 st line, 2nd line and in some cases $3^{\text {rd }}$ line medicines. This is complemented by preset medicines to give palliative relief to the suffering of the patients brought on by accompanying side effects of allopathy medicines.

\subsection{The data collection project of the PBHRFI - A unique platform for research Community}

The main research activity of PBHRFI consists of recording electronic data base of the treatments and response of all cases of various types of cancer and other life threatening diseases. The data base has a record of more than 30,000 cases with more than half a million visits. The data base consists of approximately 60 cancer types by site, including two cases of heart cancer.

This data base is active since 2002 and is epicentre to researchers from premiere institutions throughout the world. PBHRI has collaborated with researchers from the National Cancer Institute of the United States with the desire to collect more data and use the information to better the efficacy of homoeopathy medicines as well as to fine tune Banerji protocols.

\subsection{Cancer treatment outcomes at PBHRF with the Banerji Protocols}

Overall aggregate retrospective data of 18 years during the period of 1990-2008, collected on over 30,000 patients with all varieties of cancer treated reveals that $21 \%$ of the cancers completely regressed, and $23 \%$ were improved or stable. (Figure 1) 


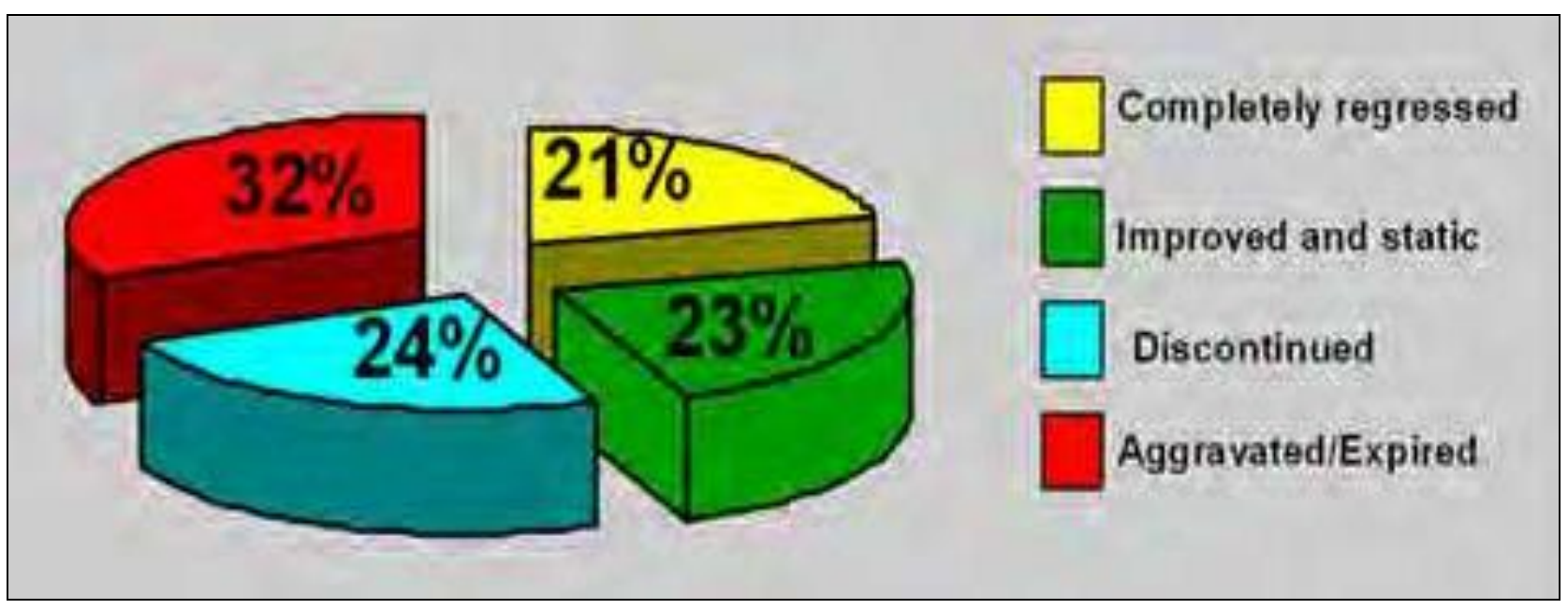

Fig 1: Results of Treatment of 30,288 Malignant Tumor Cases $(1990-2008)^{[41] .}$

Retrospective data collected over a one-year period (August 2006-August 2007) on 1132 patients treated for lung, brain and esophageal cancer showed that complete regressions ranged from 22 to $32 \%{ }^{[9]}$.

\subsection{Treatment of Brain tumor}

An extensive research at PBHRI for several years showed that the combination of homoeopathy medicines Ruta Graveleons $6 c$ and Calcarea Phosphorica $3 x$ found to be effective for the treatment of brain cancer and also for the prevention of its recurrence after surgery. It may be interesting to note that successful in vitro experiments have been done with this combination of medicines at the MD Anderson Cancer Centre in Houston USA.
The treatment of brain tumor using combination Ruta Graveleons $6 \mathrm{c}$ and Calcarea Phosphorica $3 \mathrm{x}$ is gaining popularity in India. Most of the patients are using this treatment without any form of allopathy intervention. In many other countries Homoeopathy treatment of combination medicines is being used mainly to prevent recurrence of brain tumor after surgery. PBHRFI has analysed 147 case records from different countries (India 76, USA 44, other countries including UK, Canada, Spain, Italy, Sweden, Australia, New Zealand, Netherlands, Turkey, Israel, Malaysia and Pakistan 27). The analysis showed that the combination of homoeopathy medicines have definite role to play in preventing the recurrence of brain tumors in 66.74 cases as shown in Figure 2.
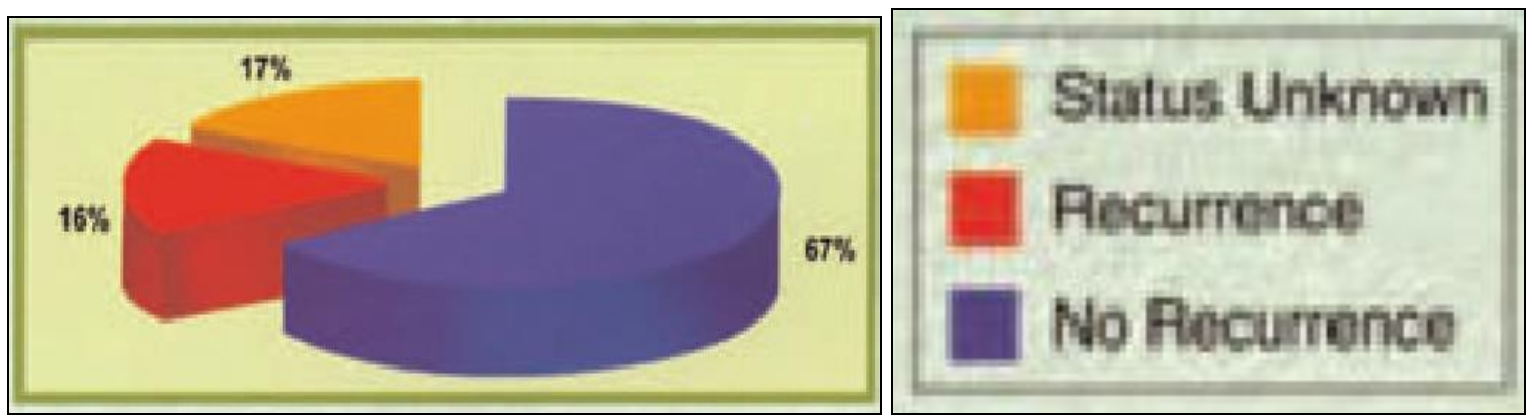

Fig 2: Outcome of treatment of brain tumor cases with Banerji protocol ${ }^{[41]}$.

\section{Homoeopathy medicines and dosage for brain tumor}

1. Ruta Graveleons 6c, one dose $=2$ drops in 1 teaspoonful of drinking water, 2 doses daily.

2. Calcarea Phosphorica $3 \mathrm{x}=2$ tablets, two doses daily
Follow up

All the patients were followed up for at least one year from the beginning of the treatment.

Outcome The outcome of the treatment is show in Figure 3 


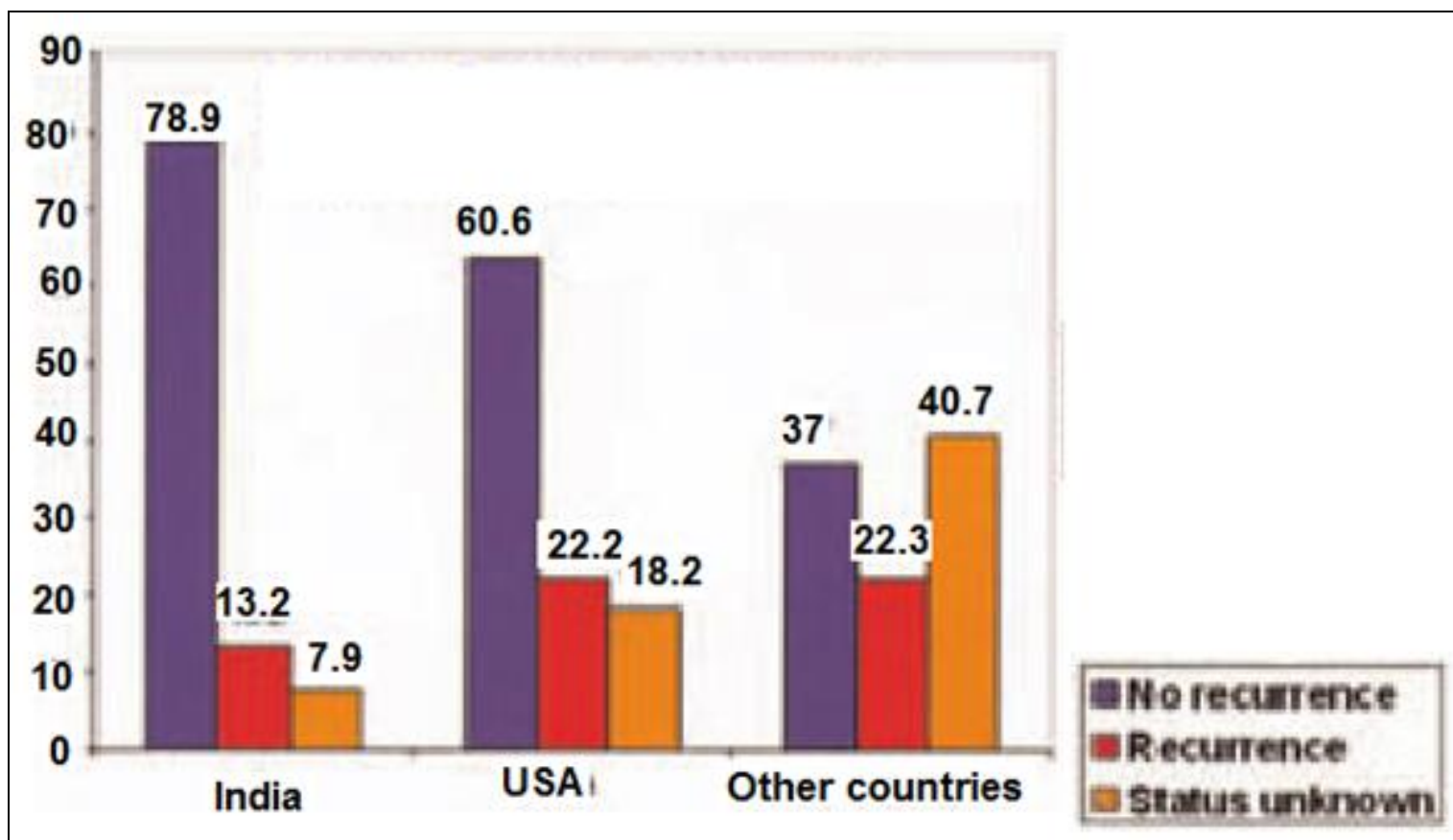

Fig 3: Outcome of the treatment of brain tumor with the Banerji Protocols in various countries ${ }^{[41]}$.

The recurrence of brain tumor after surgery is a major problem throughout the world as there is no effective treatment. The research at PBHRFI was found to be very encouraging with benefit rate as high as 66.74. However, when benefit rates for the patients from different geographical locations were compared, better improvements were obtained for patients from India and USA compared to other countries taken together. This may be due to better support system during treatment period in India and USA compared to other countries ${ }^{[41]}$.

\section{Palliative approach of homoeopathy in cancer}

Conventional cancer treatment options - including surgery chemotherapy and radiation are stressful and often result in the patient (and family) suffering; physically emotionally and financially, which negatively impacts quality of life. Chemotherapy and radiation though able to prolong the patient's life, are known to cause many side-effects including but not limited to nausea, vomiting, skin eruptions, anxiety, depression, pain and organ damage. Radiotherapy causes burning of tissues. Most treatments available for these side-effects are short-term.

Homeopathic medicines are widely used to treat side-effects of chemotherapy and radiation and non-healing wounds after surgery. Homoeopathic medicines also help in cases of neurological problems, where the patient experiences pain in the nerves. There is nothing in allopathy, except vitamins and painkillers ${ }^{[20]}$

To study the palliative approach of homeopathy; an observational study in Switzerland and Germany evaluated two cohorts (groups) of patients with cancer, one cohort was treated with homeopathic complementary therapy in addition to conventional care and one cohort was treated with only conventional care. In this study of 639 patients, researchers observed that quality of life and fatigue symptoms improved in cancer patients who received the homeopathic complementary treatment ${ }^{[42]}$.

Another randomized controlled trial evaluated homeopathy as a supplement to conventional cancer care. The patients were divided into two groups. One group received conventional treatment to prevent tumor growth and second group received homeopathic complementary therapy along with conventional treatment. This study of 285 patients revealed that patients who received the homeopathic treatment along with standard therapy had significantly better global health status and subjective well-being than those who received only conventional cancer care ${ }^{[43]}$.

A case of metastatic adenocarcinoma of the rectum, terminal squamous cell carcinoma of the cheek and carcinoma of the larynx received homeopathic medicines prescribed on constitutional grounds relieved the patient's symptoms markedly ${ }^{[44]}$.

\subsection{Palliative approach after radiotherapy}

A randomised controlled trial was conducted by Kulkarni to find the efficacy of Homoeopathy in decreasing the severity of radiotherapy-related adverse effects. Patients were randomly divided into three groups. One group was given Causticum 30, a second group was given Cobaltum 30 and the third group received placebo. Both the medicines showed decreasing symptoms of radiation reaction ${ }^{[45]}$.

In another study, effectiveness of homoeopathic medicines was investigated for radiotherapy-induced skin reactions in breast cancer patients. Patients were randomly divided in to two groups. One group received homoeopathic treatment that consisted of X-ray $15 \mathrm{cH}$ and Belladonna $7 \mathrm{cH}$ while the other group received placebo. A conventional topical medication containing fluocortolone was also administered to both groups. Homoeopathic treatment showed a temporary benefit regarding decreasing hyper pigmentation of skin. However, results became insignificant at the end of follow-up ${ }^{[45]}$.

A study with 254 participants proved that topical application of Calendula proved effective as compared to conventional Trolamine cream in radiotherapy-induced dermatitis ${ }^{[46]}$.

\subsection{Palliative approach after chemotherapy}

A preliminary, non-randomised controlled clinical trial tested the efficacy of homoeopathic medicine Traumeel S on stomatitis (inflammation of mucous membrane of mouth) 
occurred due to chemotherapy after autologous (cell or tissue obtained from the patient) or allergenic (being genetically different while belonging to same species) stemcell transplantation. A total of 20 patients including children and teenagers received Traumeel $S$ and were compared with control group of seven patients having similar stages of cancer and same age groups. The efficacy was determined by the pain level. The pain level was significantly lower in homoeopathy group compared to control group ${ }^{[47]}$.

\subsection{Palliative approach for breast cancer}

A study was conducted to find the effectiveness of homoeopathic medicines on hot flushes in breast cancer survivors. The patients included in the trial had a history of breast cancer Stage I-III, and had completed breast cancer treatment either surgical, radiation or chemotherapy and had a history of at least 3 hot flushes/day

\section{All the patients were randomised in to three different groups}

Group 1: Patients receiving individualized single homoeopathic medicine that can be one of these: Calcarea Carbonica, Kali Carbonicum, Lachesis, Sepia or Sulphur.

Group 2: Patients receiving combination medicine 'Hylands Menopause' consisted of Lachesis, Sanguinaria, Canadensis and Amyl nitrate.

\section{Group 3: Patients receiving placebo}

Sub group: patients receiving Tamoxifen (conventional allopathic medicine)

The efficacy was assessed regarding decreasing number and severity of hot flushes.

Both Homoeopathy groups showed significant improvement in quality of life score compared to placebo. In a subgroup of patients receiving Tamoxifen, was found to be more effective in reducing hot flushes compared to homoeopathic medicines and placebo ${ }^{[48]}$.

A pilot study was carried out to find the effectiveness of Homoeopathy in breast cancer survivors having estrogen withdrawal clinical features i.e. hot flushes, fatigue, anxiety, and depression. In this double-blind random clinical trial, patients having more than three hot flushes per day were selected. All the patients were divided randomly in two groups to receive either homoeopathic medicines or placebo.

Individualised homoeopathic medicines were prescribed to homoeopathic treatment group which included Arnica, Belladonna, Carcinocin, Natrum Muriaticum, Sepia and Sulphur. The efficacy was assessed by activity score (Primary outcome measures) and hot flushes frequency and severity (secondary outcome measures). No significant difference was observed between intervention and placebo group regarding both primary and secondary outcome measures at follow-up ${ }^{[49]}$.

\subsection{Palliative approach for non-small cell lung cancer (NSCLC)}

A group of 16 researcher from Medical University of Vienna, clinical division of oncology, Institute of Homoeopathic research and various other organizations evaluated the possible effects of palliative homeopathic treatment compared with placebo and control in patients with stage IV NSCLC, with respect to quality of life (QoL) in the two randomized groups and survival time in all three groups. After the treatment patients visited the outpatients' centres of Institute of homoeopathic research, every 9 weeks for observation.

150 patients with stage IV NSCLC were included in the study; 51 received individualized homeopathic remedies, 47 placebo and 52 control patients without any homeopathic treatment were observed for survival only. Different homeopathic remedies used were mainly of plant, mineral, or animal origin.

Quality of life and symptom scales showed significant improvement in the homeopathy group when compared with placebo after 9 and 18 weeks of homeopathic treatment. Average survival time was significantly longer in the homeopathy group (435 days) versus placebo and control (257 days).

A higher quality of life might have contributed to the prolonged survival. The study suggests that homeopathy positively influences not only the quality of life but also survival. Further studies including other tumor types are necessary ${ }^{[50]}$.

\section{Different studies reporting homeopathic approach for cancer care}

In 2011, the Swiss government published a report on preclinical research such as botanical studies, animal studies, and in vitro studies with human cells. After assessing the evidence from basic science research and high-quality clinical studies, it was concluded that homeopathic remedies seem to induce cellular effects as well as changes in living organisms.

The report also mentioned that 20 of the 22 systematic reviews of clinical research, testing homeopathic medicines, established a trend in favour of homeopathy. The authors concluded that homeopathic treatments should be reimbursed by Switzerland's national health insurance program, and the Swiss government accepted this recommendation ${ }^{[51]}$.

Because of the scarcity of knowledge about the efficacy of many Complimentary alternative medicines (CAM)/Complimentary integrative medicines (CIM) therapies used in cancer care, the National Cancer Institute (NCI, USA) has initiated "The Best Case Series Program" to encourage CAM practitioners to submit their data on patients over a period of 20 years, that had significant tumour reduction in response to an alternative modality for cancer treatment. Each case was reviewed against the rigorous standards of evidence of novel conventional cancer therapies ${ }^{[52,53]}$.

Prasanta Banerji and Pratip Banerji the researchers from the P Banerji Homeopathic Research Foundation India (PBHRFI) in Kolkata submitted data to this NCI program. These researchers developed a method of using homeopathic medicines through prescribing specific remedies for specific malignancies. They followed 17,324 patients with malignant tumors who were treated at PBHRFI between 1990 and 2005. In $19 \%$ of the patients, the malignant tumors completely regressed after homeopathic treatment, and $21 \%$ of the patients were the same or improved after homeopathic treatment ${ }^{[9]}$.

After rigorous evaluation of the findings of PBHRFI; NCI concluded that there was sufficient evidence of possible efficacy to warrant further research ${ }^{[54]}$. 
Another study by PBHRFI researchers and researchers at The University of Texas MD Anderson Cancer Centre, described 15 patients diagnosed with intracranial tumours who were treated exclusively with the homeopathic remedies Ruta Graveolens 6c and Calcarea Phosphorica 3X, without additional chemotherapy or radiation. Of these 15 patients, 6 patients having glioma (a malignant tumor of the glial tissue of the nervous system) showed complete regression of the tumours ${ }^{[55]}$.

On publication of this study, many patients with brain cancer that attended the integrative medicine clinic of MD Anderson showed marked interest in integrating this treatment into their conventional care ${ }^{[56]}$.

A Laboratory study conducted at MD Anderson cancer centre revealed that four ultra diluted remedies (Carcinosin, Phytolacca, Conium, and Thuja) exerted preferential cytotoxic effects against breast cancer cells, causing cell cycle delay and apoptosis without affecting the normal mammary epithelial cells.

Another observation that caused increased interest involved a comparison of the cytotoxic effect of the homeopathic remedies to Paclitaxel, a commonly used chemo therapeutic drug for breast cancer. In this study, the cytotoxic effect of two of the remedies, Carcinosin and Phytolacca, appeared to have similar cytotoxic effect to the activity of Paclitaxel on the breast cancer cells. The homeopathic remedies did not affect the normal mammary epithelial cells ${ }^{[57]}$.

A homoeopathic medicine, Sulphur, showed anti-apoptotic effect in non-small cell lung Carcinoma cells ${ }^{[58]}$.

A male with lung cancer, a woman with leiomyosarcoma and a child with an astrocytoma were treated with a homoeopathic approach of carcinogen-induced apoptosis. Ultra-low doses of carcinogens were administered for 2-3 months and showed complete remission of cancer and increased life extension ${ }^{[59]}$. Lycopodium Clavatum 5C and $15 \mathrm{C}$ administration showed anticancer effects on human cervical cancer by causing cell death through apoptosis in cancer cells ${ }^{[60]}$.

\section{Homoeopathy and survival}

There is little research on long-term survival of patients who used homeopathic care during cancer treatment.

At the Medical University of Vienna in Austria, researchers collected survival data on 538 patients who used homeopathy in addition to conventional cancer care. The study included patients with glioblastoma, lung cancer, cholangiocellular carcinoma, pancreatic adenocarcinoma, metastatic sarcoma, and metastatic renal cell carcinoma. Overall survival was compared with expert's predictions of survival outcomes by specific cancer type. The researchers found that these patients survived longer than the expected across all observed cancer types. Although the results were promising, the authors were cautious about the findings owing to the small sample size and limited data about specific treatment characteristics. The authors emphasized the need for further study of homeopathic care in cancer patients ${ }^{[20]}$.

\section{Conclusion}

Despite advances in allopathy medicines in cancer care, patients continue to experience agony during cancer treatment and afterwards. As a result, patients use complementary alternate medicines (CAM) such as homoeopathy to minimize the side effects of surgery, chemo and radiation therapies.

Limited clinical research has suggested that homeopathic remedies appear to cause cellular changes in some cancerous cells. In animal models, specific homeopathic remedies show an inhibitory effect on tumor development. Studies of homeopathic remedies combined with conventional cancer care show that these remedies reduce the burdens of side effects of allopathic medicines thus improving quality of life and possibly improve survival of cancer patients. Data from several sources suggest that cancer patients throughout the world are increasingly using homeopathy as complementary alternate medicines. These findings warrant comprehensive clinical studies to determine the effects of homeopathy on cancer and patient survival. There is a need to keep an open mind to new possibilities of homoeopathy for cancer care that in the past were considered questionable.

\section{References}

1. Ahmet Unlu, Onder Kirka, Mustafa Ozdogan. Homoeopathy and cancer. J Oncological Sciences. 2017;3:77-80

2. Ernst E, Kaptchuk TJ Homeopathy revisited. Arch Intern Med. 1996;156(21):2162-2164. Available from: jamanetwork.com

3. Hahnemann S. Organon of the Art of Healing. Boericke \& Tafel, 1833, 5th American edition translated from 5th German edition, Philadelphia. Through Ref 1

4. Gevitz N. Unorthodox medical theories. In: Bynum WF, Porter R (editors). Encyclopaedia of the History of Medicine, Routledge, London: Through Ref 1, 1993, 603-633,

5. Fisher P. What Is Homeopathy? An Introduction. Front Biosci. (Elite Ed): Through Ref 1 2012;4:1669-1682

6. Clarke TC, Black LI, Stussman BJ, Barnes PM, Nahin RL. Trends in the use of complementary health approaches among adults. United States 2002-2012 Natl Health Stat Rep 2015;79:1-16.

7. Black LI, Clarke TC, Barnes PM, Stussman BJ, Nahin RL. Use of complementary health approaches among children aged 4-17 Years in the United States. National Health Interview Survey (2007-2012). Natl Health Stat Rep 2015;78:1-19.

8. Davis RB, Kaptchuk TJ, Yeh GY. Homeopathy use by US adults: results of a national survey. Am J Public Health 2016;106:743-745

9. Prasanta Banerji, Pratip Banerji. Homeopathy: Treatment of Cancer with the Banerji Protocols. Available from: www.pbhrfi.org, interchopen.com

10. Honigberger JM. Thirty-five years in the East: Adventures, discoveries, etc., relating to the Punjab and Cashmere; in connection with medicine, botany, pharmacy. Boston, Harvard University: Through: Ref 9 $1852,1-2$.

11. Ghosh AK. A short history of the development of homeopathy in India. Homeopathy 2010;99(2):130-136.

12. Marino R. Homeopathy and Collective Health. The Case of Dengue Epidemics. International J. High Dilution Research. 2008;7:25. Through Ref 9

13. Singh P, Yadav RJ, Pandey A. Utilization of indigenous systems of medicine and homoeopathy in India. Indian J Medical Research 2005;122(2):137-142

14. World Health Organization. India: Country cooperation strategy brief 2006 Available from 
http://www.who.int/countries/ind/

15. Chakraborti D, Mukherjee SC, Saha KC, Chowdhury UK, Rahman MM, Sengupta MK. Arsenic toxicity from homeopathic treatment. J Toxicol Clin Toxicol 2003;41:963-967. Through Ref 1

16. Tumir H, Bosnir J, Vedrina-Dragojevic I, Dragun Z, Tomic S, Puntaric D. Preliminary investigation of metal and metalloid contamination of homeopathic products marketed in Croatia. Homeopathy 2010;99:183-188.

17. Braschoss A. Lycopodium similiaplex-induced acute hepatitis: A case report. Eur J Gastroenterol Hepatol 2009;2:718-719. Through Ref 1

18. WHO Library Cataloguing-in-Publication Data. Safety issues in the preparation of homeopathic medicines. Available from

http://www.who.int/medicines/areas/traditional/Homeo pathy.

19. Ernst E. Risk-free homeopathy? Schweiz Med Wochenschr 1996;126:1677-1679. Through Ref 1

20. Moshe Frenkel. Is There a Role for Homeopathy in Cancer Care? Questions and Challenges. Curr Oncol. 2015; $17: 43-48$

21. Homoeopathy for cancer Treatment. Cooperation Strategy Brief. Available from https://cancerhomoeoclinic.co.in/diseases/kidneycancer-homeopathy-treatment-clinic/

22. PubMed; search term homeopathy and cancer. Available from

http://www.ncbi.nlm.nih.gov/pubmed/?term=homeopat hy

23. Homoeopathic approach for the treatment of cancer. Saeed Ahmad, Tayyeba Rehman and Waheed Mumtaz Abbasi. Indian J Research in Homoeopathy. 2018;12(3):157-163

24. Molassiotis A, Fernadez-Ortega P, Pud D et al. Use of complementary and alternative medicine in cancer patients: A European survey. Ann Oncol 2005;16:6553. Through Ref 23

25. Koehl B, Muenstedt K, Micke O, et al. Survey of German non- medical practitioners regarding complementary and alternative medicine in oncology. Oncol Res Treat. 2014;37(1, 2):49-53. Through Ref 23

26. Bonacchi A, Fazzi L, Toccafondi A, et al. Use and perceived benefits of complementary therapies by cancer patients receiving conventional treatment in Italy. J Pain Symptom Manage 2014;47(1):26-34 Through Ref 23

27. Rossi E, Vita A, Baccetti S, Di Stefano M, Voller F, Zanobini A. Complementary and alternative medicine for cancer patients: Results of the EPAAC survey on integrative oncology centres in Europe. Support Care Cancer 2015;23(6):1795-806. Through Ref 20

28. Amri H, Anderson N, MacLaughlin WB, Esquivel S, Ives J, Jonas W, Effects of ultra-low dilutions of Sabal serrulata on human prostate cancer growth: mechanistic studies. Alternat Therapies 2009;15:S79-80. Through Ref 20

29. MacLaughlin BW, Gutsmuths B, Pretner E, et al. Effects of homeopathic preparations on human prostate cancer growth in cellular and animal models. Integr Cancer Ther 2006;5:362-372.

30. Sunila ES, Kuttan G. A preliminary study on antimetastatic activity of Thuja occidentalis L. in mice model. Immunopharmacol, Immunotoxicol
2006;28:269-80. Through Ref 20

31. Kumar KB, Sunila ES, Kuttan G, Preethi KC, Venugopal CN, Kuttan R. Inhibition of chemically induced carcinogenesis by drugs used in homeopathic medicine. Asian Pac J Cancer Prev 2007;8:98-102.

32. Biswas SJ, Khuda-Bukhsh AR. Effect of a homeopathic drug, Chelidonium, in amelioration of p-DAB induced hepatocarcinogenesis in mice. BMC Complement Altern Med 2002;2:4.

33. Es S, Kuttan G, Kc P, Kuttan R. Effect of homeopathic medicines on transplanted tumors in mice. Asian Pac J Cancer Prev 2007;8:390-394.

34. Bhattacharjee N, Pathak S, Khuda-Bukhsh AR. Amelioration of carcinogen-induced toxicity in mice by administration of a potentized homeopathic drug, natrum sulphuricum 200. Evid Based Complement Alternat Med 2009;6:65-75.

35. Korengath Preethi, Sunila Ellanghiyil, Girija Kuttan, Ramadasan Kuttan, Induction of Apoptosis of Tumor Cells by Some Potentiated Homeopathic Drugs: Implications on Mechanism of Action, Integrative Cancer Therapies 2012;11(2):172-182.

36. Surjyo Jyoti Biswas, Surajit Pathak, Nandini Bhattacharjee, Jayanta Kumar Das, Anisur Rahman Khuda-Bukhsh. Efficacy of the Potentized Homeopathic Drug, Carcinosin 200, Fed Alone and in Combination with Another Drug, Chelidonium 200, in Amelioration of $\mathrm{p}$ Dimethylaminoazobenzene-Induced Hepatocarcinogenesis in Mice. J. Alternative and Complementary Medicine. 2005;11:839-854.

37. Shagun Arora, Ayushi Aggarwal, Priyanka Singla, Saras Jyoti, Simran Tandon. Anti-proliferative effects of homeopathic medicines on human kidney, colon and breast cancer cells. Homeopathy 2013;102:274-282.

38. Tiantian She, Like Qu, Lixin Wang, Xingxin Yang, Shuo $\mathrm{Xu}$, Junnan Feng et al. Sarsaparilla (Smilax Glabra Rhizome) Extract Inhibits Cancer Cell Growth by $S$ Phase Arrest, Apoptosis, and Autophagy via Redox-Dependent ERK1/2 Pathway. Cancer Prev Res. 2015;8(5):464-74.

39. Gilles Freyer, Benoit You, Stephanie Villet, Sophie Tartas et al. Open-label uncontrolled pilot study to evaluate complementary therapy with Ruta Graveolens $9 \mathrm{c}$ in patients with advanced cancer. Homeopathy. 2014;103:232-238.

40. Sen Pathak, Asha S, Multani, Pratip Banerji, Prasanta Banerji. Ruta 6 selectively induces cell death in brain cancer cells but proliferation in normal peripheral blood lymphocytes: A novel treatment for human brain cancer. International J Oncology 2003;23:975-982.

41. Prasanta Banerji, Pratip Banerji. A new method of treatment of brain tumors with Ruta and Calcarea Phosphorica. Available from Pbhrfindia.org

42. Rostock M, Naumann J, Guethlin C, Guenther L, Bartsch HH, Walach H. Classical homeopathy in the treatment of cancer patients-a prospective observational study of two independent cohorts. Cancer 2011;11:19.

43. Frass M, Friehs $\mathrm{H}$, Thallinger $\mathrm{C}$, et al. Influence of adjunctive classical homeopathy on global health status and subjective well-being in cancer patients-a pragmatic randomized controlled trial. Complement. Ther Med 2015;23(3):309-17.

44. Rajendran ES. Homeopathy as a supportive therapy in cancer. Homeopathy 2004;93:99-102. 
45. Kulkarni A. Radiation protection by use of homoeopathic medicines. Hahnemannian Homoeopathic Sandesh 1988;12:20-23.

46. Pommier P, Gomez F, Sunyach MP, D'Hombres A, Carrie C, Montbarbon X. Phase III randomized trial of Calendula officinalis compared with trolamine for the prevention of acute dermatitis during irradiation for breast cancer. J Clin Oncol 2004;22:1447-53.

47. Oberbaum M, Yaniv I, Ben-Gal Y, Stein J, Ben-Zvi N, Freedman LS. A randomized, controlled clinical trial of the homeopathic medication Traumeel $\mathrm{S}$ in the treatment of chemotherapy-induced stomatitis in children undergoing stem cell transplantation. Cancer 2001;92:684-90.

48. Jacobs J, Herman P, Heron K, Olsen S, Vaughters L. Homeopathy for menopausal symptoms in breast cancer survivors: a preliminary randomized controlled trial. J Altern Complement Med. 2005;11:21-27.

49. Thompson EA, Montgomery A, Douglas D, Reilly D. A pilot, randomized, double-blinded, placebo-controlled trial of individualized homeopathy for symptoms of estrogen withdrawal in breast-cancer survivors. J Altern Complement Med 2005;11:13-20.

50. Michael Frass, Maximilian Hochmair, Peter Lechleitner et al. Homeopathic Treatment as an Add-On Therapy May Improve Quality of Life and Prolong Survival in Patients with Non-Small Cell Lung Cancer: A Prospective, Randomized, Placebo-Controlled, DoubleBlind, Three-Arm, Multicenter Study. Oncologist 2020;25:1930-1955.

51. Bornhoft G, Matthiessen PF. Homeopathy in healthcare: effectiveness, appropriateness, safety, costs. Goslar: Springer 2011. Through Ref 20.

52. National Cancer Institute. NCI Best Case Series Program. Available from http://cam.cancer.gov/best case series program.html.

53. Olaku O, Zia F, Santana JM, White JD. The National Cancer Institute Best Case Series Program: a summary of cases of cancer patients treated with unconventional therapies in India. Integrated Cancer Ther 2013;12(5):385-392.

54. Banerji P, Campbell DR, Banerji P. Cancer patients treated with the Banerji protocols utilising homoeopathic medicine: a Best Case Series Program of the National Cancer Institute USA. Oncol Rep 2008;20:69-74.

55. Pathak S, Multani AS, Banerji P, Banerji P. Ruta 6 selectively induces cell death in brain cancer cells but proliferation in normal peripheral blood lymphocytes: a novel treatment for human brain cancer. Int $\mathrm{J}$ Oncol 2003;23:975-82.

56. Frenkel M, Cohen L, Peterson N, Swint K, Palmer L, Bruera E. Integrative medicine consultation service in a comprehensive cancer centre: findings and outcomes. Integr Cancer Ther 2010;9(3):276-83.

57. Frenkel M, Mishra BM, Sen S et al. Cytotoxic effects of ultra diluted remedies on breast cancer cells. Int $\mathbf{J}$ Oncol 2010;36(2):395-403.

58. Saha S, Bhattacharjee P, Guha D, Kajal K, Khan P, Chakraborty $\mathrm{S}$ et al. Homoeopathic Sulphur induces apoptosis in non-small cell lung carcinoma. Int $\mathrm{J}$ Oncol 2015;47:573-582.

59. Montfort H. A new homeopathic approach to neoplastic diseases: From cell destruction to carcinogen-induced apoptosis. Br Homeopath J 2000;89:78-83.

60. Samadder A, Das S, Das J, Paul A, Boujedaini N, Khuda-Bukhsh AR et al. The potentized homeopathic drug, Lycopodium clavatum (5C and 15C) has anticancer effect on hela cells in vitro. J Acupunct Meridian Stud 2013;6:180-187. 